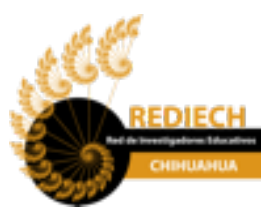

Red de Investigadores Educativos Chihuahua A.C. Chihuahua, México www.rediech.org

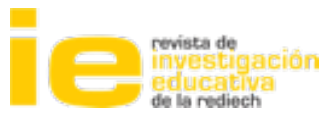

ISSN: $2007-4336$

ISSN-e: $2448-8550$

http://www.rediech.org/ojs/2017/index.php/ie rie rediech/index

Pavel Roel Gutiérrez Sandoval

Evangelina Cervantes Holguín

Iskra Rosalía Gutiérrez Sandoval

2019

\title{
Innovación y experiencias creativas de matemática educativa en escuelas secundarias desde la pedagogía del malabarismo
}

IE Revista de Investigación Educativa de la REDIECH, 10(18), pp. 65-78. https://dx.doi.org/10.33010/ie_rie_rediech.v10i18.214

\section{(c) $(1)(\Theta$}

Esta obra está bajo licencia internacional

Creative Commons Reconocimiento-NoComercial 4.0. 


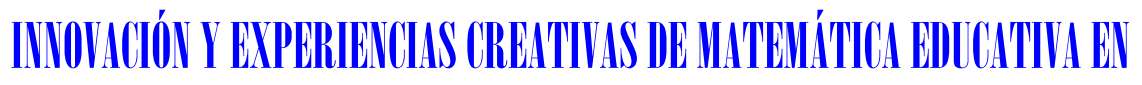

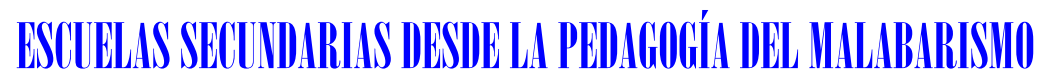

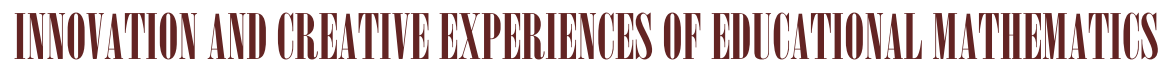

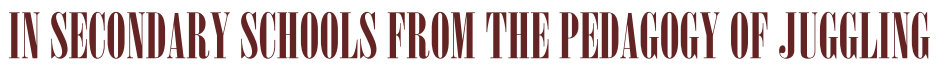

\author{
GUTIÉRREZ SANDOVAL Pavel Roel \\ CERVANTES HOLGUÍN Evangelina \\ GUTIÉRREZ SANDOVAL Iskra Rosalía
}

Recepción: agosto 1 de 2018 | Aprobado para publicación: noviembre 27 de 2018

DOI: https://dx.doi.org/10.33010/ie_rie_rediech.v10i18.214

\section{Resumen}

El entrenamiento en malabares duró seis meses con el método Rehoruli (grupo 1) e Ingalese (grupo 2) con 47 adolescentes de secundaria y se mantuvieron dos grupos control sin entrenamiento. Ambos grupos experimentales tuvieron mejores resultados que los grupos control en la calificación final de la clase de matemáticas y en el rendimiento en tareas de rotación mental e identificación de vértices, lados y caras planas en figuras tridimensionales. Además, la aplicación Juggling Lab mejoró la identificación y ejecución de patrones numéricos observados.

Pável Roel Gutiérrez Sandoval. Profesor de tiempo completo de la Universidad Autónoma de Ciudad Juárez, Chihuahua, México. Es responsable del curso de posgrado "Taller de arte feminista: análisis y creación desde las artes escénicas contemporáneas en el Programa de Maestría en Estudios Interdisciplinarios de Género", integrado al PNPC de Conacyt. Es miembro del Sistema Nacional de Investigadores SNI-Nivel I. Es responsable de la Unidad Técnico-Experimental en Fonoaudiología, Semántica-Léxica y Didáctica Musical de la UACJ-Conacyt 2015. Además, coordina el "Diplomado de especialidad en educación musical aplicada" y el Programa de Talleres Artísticos. Correo electrónico: pavel.gutierrez@uacj.mx. ID: http://orcid.org/0000-0003-0437-1549.

Evangelina Cervantes Holguín. Profesora de tiempo completo de la Universidad Autónoma de Ciudad Juárez, Chihuahua, México. Está adscrita al Programa de Maestría en Investigación Educativa Aplicada perteneciente al PNPC de Conacyt. Es miembro del Sistema Nacional de Investigadores del Conacyt SNI-Nivel I, asesora de la Unidad Técnico-Experimental e Instructora del "Diplomado de especialización en educación musical aplicada". Correo electrónico: evangelina.cervantes@uacj.mx. ID: http//orcid.org/0000-0001-6980-2210.

Iskra Rosalía Gutiérrez Sandoval. Profesora de la Universidad Autónoma de Ciudad Juárez, Chihuahua, México. Es licenciada en artes visuales y maestra en estudios y procesos creativos en arte y diseño. Es instructora del diplomado y del Programa de Talleres Artísticos. Correo electrónico: iskra.gutierrez@gmail.com. ID: http://orcid.org/0000-00033949-7774. 


\author{
Palabras clave: INNOVACIÓN EDUCATIVA, EXPERIENCIAS CREATIVAS, \\ MODELO STEM, MATEMÁTICA EDUCATIVA, PEDAGOGÍA DEL \\ MALABARISMO.
}

\begin{abstract}
The training in juggling lasted six months, using the Rehoruli method (group 1) and Ingalese (group 2) with 47 high school adolescents and two control groups without training were maintained. Both experimental groups had better results than the control groups in the final grading of math class and in the performance of tasks involving mental rotation and identification of vertices, sides and flat faces in three-dimensional figures. In addition, the Juggling Lab implementation improved the identification and execution of observed numerical patterns.
\end{abstract}

Keywords: EDUCATIONAL INNOVATION, CREATIVE EXPERIENCES, STEM
Model, EDUCATIONAL MATHEMATICS, PEDAGOGY OF JUGGLING.

IVTRonicciós

La National Advocates for Arts Education (NAAE, 2017) señala que la educación artística enfrenta en el siglo XXI el reto de ser tanto innovadora como creativa por una demanda en el plano de la industria en los mercados globales, lo cual se extiende más allá del empleo de la tecnología o de los productos de la investigación científica en la empresa. Al respecto, la innovación y la creatividad se refleja en la hibridación entre las mismas artes, entre las artes y la educación científica, así como de las artes con las disciplinas humanísticas. Se coincide en que incluir en la escuela un conjunto de habilidades investigativas y desarrollar una vinculación de la escuela con las industrias culturales públicas y privadas son dos de los factores determinantes para desarrollar la creatividad e impactar en los objetivos sociopolíticos, patrimoniales y pedagógicos de la educación artística.

Al respecto, el sistema educacional australiano destaca entre los países industrializados que han insertado las habilidades de innovación y creatividad en las disciplinas de la llamada educación científica basada en el modelo STEM (Science, Technology, Engineering and Mathematics) desde las adecuaciones curriculares a finales del siglo Xx (este modelo se considera de carácter flexible, integrado y aplicado para enseñar en un contexto real dichas asignaturas) (NAAE, 2017).

En este sentido, los retos de la educación científica y de la educación artística encierran las siguientes categorías de análisis: disciplina, toma de decisiones, resolución de problemas, flexibilidad, reflexión crítica, trabajo colaborativo, automatización, disociación, pensamiento en activo, pensamiento figurativo, pensamiento asociativo, emprendedurismo, innovación, creatividad, improvisación, curiosidad, imaginación, fantasía, experimentación, resiliencia, confianza, medios alternativos 
de comunicación, etcétera, por lo que la educación artística y el modelo STEM no son indistintos.

Al respecto, en 2013 la Organización para la Cooperación y el Desarrollo Económicos (OCDE) encontró una relación positiva entre artes e innovación, ya que alrededor de 54 por ciento de las y los estudiantes graduados de carreras de artes se encuentran entre las y los profesionistas más propensos a tener un alto resultado innovador -desarrollo de productos, tecnología o conocimientos- cinco años después de la graduación (citado en NAAE, 2017).

Por otro lado, la enseñanza de las artes en las escuelas de enseñanza general en Rusia en el ciclo escolar 2017-2018 insiste en los contenidos de diferentes tipos y géneros de las artes, como las artes visoespaciales -incluye las bellas artes: música, danza, pintura o escultura-, aquellas de índole decorativo y aplicado -como la arquitectura 0 el diseño de interiores- y a través de las artes sintéticas, como el teatro, el circo o el cine. De esta manera, la educación artística rusa contribuye a la formación de la identidad cultural que garantiza la motivación por el aprendizaje de las artes, así como la formación de los cimientos de la cultura artística de las y los estudiantes como parte de su cultura espiritual común; el desarrollo de la capacidad de observancia, de empatizar, de memoria visual, de pensamiento asociativo, gusto artístico e imaginación creativa, entre otros objetivos más (Adoevtseva, 2017).

Asimismo, Kreilinger (2015) señala que la mayor innovación en la educación en Europa del Este propone seis acciones curriculares: 1) tener espacios para discutir y defender el papel obligatorio y sustantivo de las artes en cualquiera de sus manifestaciones; 2) visibilizar las condiciones y sensibilizar los beneficios que tiene el empleo de las artes en su transversalización en el proceso de enseñanza-aprendizaje a través de proyectos para aprender mediante el empleo de las artes; 3 ) sistematización de las orientaciones metodológicas y guías técnicas para docentes sobre las didáctica particulares de cada una de las manifestaciones artísticas que se incluyan en los programas educativos; 4) evaluar el desempeño artístico de las y los estudiantes permitiendo a ellas/os aprender sobre sus propios resultados y sobre las manifestaciones de mejor desempeño e interés; 5) unir la educación artística con el reciente interés por la educación para la diversidad mediante la asignatura arte y cultura; $\mathrm{y}$, 6) emplear la aplicación tecnológica a la enseñanza de las artes y su relación con las disciplinas de la educación científica.

Siguiendo con lo anterior, la agencia Golant Media Ventures (2015) reconoce el papel de la tecnología en el futuro de la educación, considerando que aquello que se había mostrado en el cine de ciencia-ficción en el siglo xx, hoy las y los científicos e ingenieros ya llegaron allí. Las nuevas tecnologías se están utilizando dentro del proceso creativo, por ejemplo: los recorridos virtuales en museos, así como la participación interactiva entre el espectador con la obra artística presentada.

Por un lado, la innovación tecnológica en el campo digital es una demanda de los organismos financiadores y esta es importante para las fuentes de financiamiento -como son el gobierno, las empresas y las asociaciones civiles- al garantizar una mayor sostenibilidad de cada proyecto artístico. Por otro lado, el sector informal -reconocido como agrupaciones de artistas callejeros, de garaje o independientes- han estado generando las aplicaciones realmente innovadoras de la tecnología 
digital en el montaje escénico y en el proceso de adiestramiento artístico mediante la experimentación creativa.

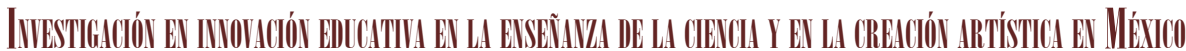

En México no hay investigación educativa experimental mediada desde las artes circenses ni enfocada a los procesos creativos y su vinculación con las disciplinas STEM. En el país existen 13 cuerpos académicos (CA) registrados en el Programa para el Desarrollo Profesional Docente (Prodep) en 12 instituciones de educación superior (IES) mexicanas: cuatro universidades, cuatro escuelas normales, un Centro de Actualización del Magisterio, un Centro Regional de Formación Docente e Investigación, una Escuela de Ciencias de la Educación y un instituto tecnológico. Los 13 cuerpos académicos centran la innovación educativa como categoría de estudio. Solamente la Universidad Autónoma de Guerrero cuenta con dos CA's que trabajan en sus proyectos de investigación la innovación educativa. En total hay 50 profesores de tiempo completo (PTC's) en los 13 CA's del país (24 hombres investigadores y 26 mujeres investigadoras). Además, cinco CA's tienen el grado en formación, cinco CA's en consolidación y tres CA's tienen el grado consolidados.

Hay 13 docentes en estos últimos tres CA's cuya obtención del grado indica que 26 por ciento de los PTC's en CA's de innovación educativa presentan evidencia a la fecha de tener trabajo colegiado alrededor de una línea de investigación sólida, trabajo en redes (inter)nacionales y generación de productos de investigación especializados. Sin embargo, solo cuatro de 50 PTC's tiene publicaciones relevantes de innovación educativa en las disciplinas del modelo STEM, pero ninguno hace investigación en y sobre innovación basada en las artes. Sus investigaciones se relacionan con las siguientes categorías: sociedad del conocimiento, procesos de formación docente, competencias para la docencia, evaluación educativa, gestión y cultura en las instituciones, prácticas curriculares, tecnologías educativas, sustentabilidad, enseñanza de las ciencias y las matemáticas o educación física. Destaca el CA 201 Desarrollo Profesional del Docente de Matemáticas e Innovación Educativa de la Universidad Autónoma de Guerrero con grado en consolidación e integrado por tres mujeres investigadoras y un hombre investigador que buscan implementar resultados de investigación que han girado en torno a la construcción social del conocimiento matemático, el dominio afectivo y la innovación educativa en medios que promuevan el desarrollo profesional docente, principalmente en profesores de matemáticas.

Del análisis del estado de la investigación que se hizo rastreando las publicaciones con impacto en el modelo STEM del CA 201, destaca la Dra. María Esther Magali Méndez Guevara con investigaciones en matemática educativa en la línea de resignificación de la modelación matemática en la escuela y como formadora en varios espacios: el Diplomado Nacional sobre las Matemáticas y su Enseñanza en la Escuela Primaria por la Universidad Pedagógica Nacional (UPN); los laboratorios virtuales de ciencias en planteles de educación media-superior Conalep, Cobach y CeCyteg; la especialización de alto nivel para docentes de matemáticas de educa68 ción secundaria ofrecida por la SEP; y el Diplomado Desarrollo de Estrategias de 
Aprendizaje para las Matemáticas para Profesores de Bachillerato ofrecido por la SEP. En el mismo tenor, el Dr. José Efrén Marmolejo Valle con publicaciones sobre las tecnologías aplicadas a la enseñanza de las matemáticas. Así como de la Dra. Marcela Ferrari Escolá sobre los logaritmos en el discurso matemático escolar y la construcción del conocimiento matemático. Además, el trabajo de la Dra. Magdalena Rivera Abrajan sobre el estudio de las creencias, las emociones, las identidades las actitudes, los valores y la motivación de estudiantes y profesores hacia las matemáticas, su enseñanza y su aprendizaje.

Siguiendo con lo anterior, se analizan las categorías creación, artes y educación, logrando encontrar seis CA's reconocidos por Prodep con un total 19 PTC's (ocho hombres investigadores y 11 mujeres investigadoras). Entre estos, hay un CA en formación, tres CA's en consolidación y dos CA's consolidados. Los seis CA's consideran la creación como una competencia para la formación de las y los estudiantes de las artes. En términos específicos de la manifestación música, la creación está relacionada directamente con la interpretación, la improvisación, la composición y es parte de los nuevos modelos de la educación musical. Cabe mencionar que ninguno de estos seis CA's utiliza la categoría innovación educativa directamente. Destaca el CA 721 Enseñanza de las Ciencias y las Artes en Ambientes Virtuales de la Universidad de Guadalajara, con grado en consolidación, está integrado por tres mujeres investigadoras que trabajan el diseño, experimentación y evaluación de métodos, estrategias y recursos para la enseñanza de disciplinas científicas y artísticas. Del análisis del estado de la investigación que se hizo rastreando las publicaciones del CA 721 se concluye que no hay publicaciones sobre las disciplinas del modelo STEM.

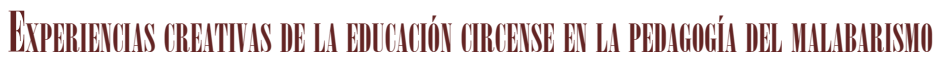

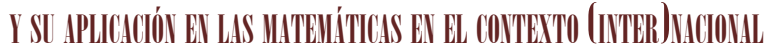

Las disciplinas y técnicas de la educación circense definidas por la Escuela Nacional de Circo en Francia son: 1) equilibrio estático o dinámico;2) manipulación de malabares en el aire; 3) actividades áreas con arneses u otros accesorios; 4) actor de circo con las técnicas clown, juego teatral, danza, mimo o máscaras; y, 5) acrobacias en el suelo sin accesorios, contorsionismo o con accesorios (citado por Guzmán, Moral y Torres, 2015). Se coincide en que las artes circenses pueden ser de bajo 0 alto riesgo.

Este apartado analizó las experiencias creativas en el empleo de las artes circenses con referencia a la disciplina malabares y la matemática educativa. Como punto de partida se reconoce al circo como una de las artes escénicas de mayor creatividad, lo cual va acorde con las metas educativas 2021 para los países iberoamericanos, pues la enseñanza de las artes es una pieza clave de la agenda política de la próxima década al propiciar creatividad e innovación en las escuelas (Ruiz y Mota, 2009).

Por lo tanto, la enseñanza de las artes circenses se está produciendo desde múltiples metodologías y no solo dentro de las grandes compañías de circo con una enseñanza familiar como se hacía tradicionalmente. Tampoco se produce dentro de las escuelas de circo legalmente establecidas nacional o estatalmente después de la segunda mitad del siglo XX, sino que hoy algunas universidades, hospitales, programas 
públicos de circo social $\mathrm{y} / 0$ centros deportivos ofrecen a una diversidad de usuarios entrenamiento en las disciplinas de circo a través de diplomados, seminarios, talleres, workshops y otros cursos impartidos por investigadores universitarios, educadores, médicos, artistas circenses, cirqueros de las calles o barrios, ingenieros e incluso matemáticos (Turner, Dubois y Moris, 2016).

Como puede observarse con la cita anterior, ya se ha superado la crisis económica que atravesó el sector artístico circense de 1950-1990, caracterizada por la precarización laboral, bajos salarios, nomadismo y horarios irregulares, cuyo declive era provocado por la desafección del público al circo tradicional. Sin embargo, en las últimas tres décadas las artes circenses se han revolucionado, convirtiéndose en punta de lanza de la creatividad y la innovación artística. Destaca la definición del campo profesional educación circense, cuyas temáticas han sido incluidas en las clases de educación artística, educación física o matemáticas de algunos centros escolares (GPECF, 2012).

Price (2012) señala que, aunque los estudios de educación circense se fortalecen con evidencia científica de todo tipo, el uso de actividades circenses en la educación es poco documentado y, por ende, escaso. Asimismo, Alcántara (2012) señala que la formación del educador circense tiene que romper la frontera entre el artista, el defensor social y el educador tradicional. Al respecto, Guzmán, Moral y Torres (2015) encuentran que la educación circense ofrece flexibilidad conceptual y operativa para apoyar los objetivos del programa de educación básica y educación especial, aunque las propuestas con frecuencia se encuentran en las etapas de educación primaria y secundaria, dejando olvidada la educación preescolar.

Jansen, Lange y Heil (2011) sostienen que la investigación sobre la educación circense aplicada consiste en llevar a la práctica las teorías sobre la relación que tiene el entrenamiento en las artes de circo con las funciones cognitivas ejecutivas de control central en el cerebro, al tiempo que se realizan actividades de movimiento, así como las funciones modales vinculadas al desarrollo motor. Al respecto, Palominos et al. (2018) proponen una metodología para la enseñanza de técnicas de malabarismo en el contexto escolar que apoye el desarrollo artístico, cognitivo, motriz y socioafectivo mediado por el entrenamiento de las capacidades de fuerza, resistencia, velocidad y flexibilidad, así como por el estiramiento o calentamiento funcional para una correcta movilidad articular y elasticidad muscular.

Las malabares son una disciplina de las artes circenses cuya técnica consiste en manipular objetos de diferentes formas, tamaños, pesos y cantidades de manera artística, deportiva, matemática o recreativa. Según la clasificación por objetos del malabarismos, tenemos: malabares de lanzamiento (pañuelos, pelotas, clavas o aros), malabares de equilibrio dinámico (monociclo, rolla-bolla o pelota de equilibrio) y estático (con alguna parte del cuerpo desplazamos su base para mantener el equilibrio), malabares giroscópicos (plato chino, diábolo, golo y hula), malabares de contacto (cajas, sombreros y rolling balls), malabares swing (consisten en circunducciones con las extremidades superiores usando como eje el hombro, codo y/o muñeca, combinadas con rotaciones del objeto en diferentes planos, ya sean clavas, banderas 0 pajaritos), malabares no convencionales (shaker, figuras geométricas, dapo o pañuelo 70 de spinning y buegeng) (Palominos et al., 2018). 
En este sentido, Echeverry y Audor (2013) señalan que los juegos malabares, además de definirse como una manipulación de objetos ejecutando movimientos precisos en el aire, tienen algunos aspectos matemáticos, denominados patrones de malabarismo simples o complicados. Los números son importantes para la ejecución; existe una razón de permanencia de los objetos en la mano. Cuando se tiene durante más tiempo la bola en la mano, el jugador tiene más control sobre el lugar específico en el que se lanzará la bola restante. Cuando la razón de permanencia es pequeña, es decir, el tiempo de permanencia de la bola en la mano es relativamente corto, el jugador tiene más tiempo para corregir la posición de las manos y aumenta la posibilidad de tener más objetos en el aire.

Respecto al aprendizaje de los patrones de las malabares, Engström, Leskelä y Varpanen (2013) indican que las malabares apoyan el desarrollo de la libre exploración, el descubrimiento guiado y la resolución de problemas. Además, el modelo pedagógico se basa en la enseñanza recíproca en el que las y los aprendices que ejecutan bien un determinado ejercicio lo enseñen a un compañero (favorece un modelo de coeducación).

Además, Czedli (2013) señala que entre los patrones simples están aquellos conocidos como de latido constante, que ocurren igualmente espaciados en el tiempo por semejarse al segundero del reloj. Otra característica es que las figuras de malabares son periódicas [ $\infty$ ], la altura del lanzamiento será medida en segundos que el objeto permanecerá en el aire, así como los segundos de reposo que el objeto permanece en las manos. Esto da como resultado la secuencia de altura en cada patrón de malabarismo.

En 1981, Paul Klimek crea en California, Estados Unidos, las notaciones transposicionales que permiten conocer los patrones de malabares posibles mediante secuencias de números que las conforman. Luego, estas fueron desarrolladas en años posteriores por el estadunidense Bruce Tiemann y por el inglés Michael Dayle. Estas notaciones transposicionales representan el orden del lanzamiento de los objetos a través del tiempo, la altura a la cual llegan y con qué mano -derecha o izquierdase atrapan. Cada número define la cantidad de tiempo que tardará el objeto en ser nuevamente lanzado (mientras más alto sea el número, más alto será el lanzamiento). El promedio de los números del patrón debe ser un número entero para que el patrón de malabares sea válido. Por ejemplo, un patrón 441 se ejecuta con 3 objetos, ya que al sumar $4+4+1=9$ y esto se divide en la cantidad de dígitos que son: $9 / 3=3$ objetos. Además, al trazar una línea del tiempo de cada patrón puede conocerse si este es válido o no (Palominos et al., 2018).

Al respecto, Polster (2015) señala que el matemático malabarista más antiguo que conocemos es Abū Sahl al-Qūhī de Oriente Medio, un musulmán y persa que vivió en el siglo $\mathrm{x}$ d.C. Antes de hacerse famoso como matemático al resolver varios problemas geométricos, hizo juegos malabares con botellas de vidrio en el mercado de la ciudad de Bagdad en Irak. Fue el primero en describir una secuencia de cadenas finitas de los números que muestran los intercambios de sitios que tienen las bolas que se lanzan durante una secuencia específica con $[1,2, \ldots n]$ bolas utilizadas por el malabarista experto. Además, utilizaba con frecuencia las malabares como didáctica para explicar las trayectorias cónicas (círculos, elipses, parábolas e hipérbolas). 
Fue hasta 1985 cuando matemáticos y malabaristas aficionados, como Bengt Magnusson, Bruce Tiemann, Paul Klimak, Adam Chalcraft, Mike Day y Colin Wright, comenzaron a desarrollar el lenguaje matemático a partir de la observación de los patrones de las malabares. A la fecha, hay simuladores de malabares en red, como el Juggling Lab diseñado por Jack Boyce. Estos simuladores hacen posible comprender los patrones malabares con cuatro - patrón par de bolas que siguen una trayectoria circular- y cinco bolas - patrón impar de bolas que sigue una trayectoria

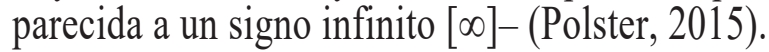

Ayyer, Bouttier y Corteel (2015) sostienen que entre las malabares y las matemáticas destacan la exploración que realizó Polster con apoyo de la geometría algebraica. Sin duda, el modelo simple propuesto por Warrington parte de los principios físicos y ha tenido una generalización entre las estrategias didácticas propuestas por Engström, Leskelä y Varpanen, quienes trabajan con pelotas y lanzamientos a una altura arbitraria para que los aprendices comprendan que el espacio para malabares no es infinito, sino que cada usuario tiene que observar y conocer sus límites en función a la fuerza, la duración y el patrón de malabares que se esté utilizando. Existe evidencia teórica que sustenta los malabarismos y cuya complejidad corresponde al número de objetos que se utilizan en el lanzamiento. La dificultad se eleva cuando se trabaja con una mayor cantidad de objetos y en la medida en que dos o más objetos son lanzados al mismo tiempo. Esto último ha sido ampliamente estudiado por Ehrenborg y Readdy (citados por Ayyer, Bouttier y Corteel, 2015).

En términos matemáticos (algebraicos), los patrones de malabarismo siguen el modelo propuesto por Claude Shannon $(\mathrm{f}+\mathrm{d})^{*} \mathrm{~h}=(\mathrm{v}+\mathrm{d})^{*} \mathrm{n}$, donde: [f] es el tiempo que permanece una bola en el aire, [d] tiempo que una bola se sostiene en la mano, [h] número de manos que intervienen, [v] tiempo en que una mano está vacía y [n] número de bolas en el juego de malabares. A partir de lo anterior es posible despejar la ecuación para cada variable según se disponga de los datos que se mencionan. $\mathrm{La}$ experimentación matemática con las malabares ha hecho posible introducir nuevos patrones para que las y los malabaristas intenten, creen y definan estéticamente nuevas figuras (citado por Ouellette, 2013).

Butler et al. (2015) sostienen, en un modelo matemático básico, que la conexión del patrón de malabares es en el momento [i]; el objeto se lanza de modo que desciende en unidades de tiempo lo que daría como ecuación $\mathrm{T}=\mathrm{i}+\mathrm{ti}(\bmod \mathrm{tn})$. $\mathrm{La}$ secuencia [ti] es periódica de modo que el patrón expandido es en realidad [t1, t2 hasta tn]. Otra precisión es que la secuencia [T] es una secuencia de intercambios de sitios donde nunca sucede que dos bolas bajen al mismo tiempo, pues se rompería el patrón. Una secuencia de malabares es continua ( 3,4 y 5$)$. Además, el número de bolas $[\mathrm{b}]$ necesarias para realizar la secuencia $[\mathrm{T}]$ es el promedio:

$$
b=\frac{1}{n} \sum_{i=1}^{n} t i
$$

Además, el número de bolas para cada secuencia de malabarismo seria $\mathrm{b}^{\mathrm{n}}$. Por otro lado, Ehrenborg y Readdy diferencian entre secuencias de tiempo y secuencias de 72 orden en los patrones de malabarismo; esto sirve para poder distinguir figuras malaba- 
res de uno o más ciclos de transición entre figuras. Es posible aumentar el número de bolas atrapadas y luego arrojarlas al mismo tiempo, lo que se conoce como malabares múltiples. Por otro lado, si se conoce la cantidad y el orden de las bolas que serán lanzadas respecto a su tiempo-altura, entonces es posible definir matemáticamente el patrón de malabares en función a la permutación. Por ejemplo, en las malabares con tres bolas -una en la mano izquierda y dos en la mano derecha-, la única bola que cambia de posición es la lanzada por la mano izquierda hacia la mano derecha y una de las dos bolas de la mano derecha es desplazada a la mano izquierda para dar continuidad a la secuencia. Por último, la ecuación matemática según el número de lanzamientos, bolas y cruces o conexiones en un solo ciclo quedaría:

$$
\left\{\frac{n}{k}\right\}_{m}=\frac{(-1)^{k}}{k !} \sum_{i=m}^{k}(-1)^{i}\left(\begin{array}{l}
k \\
i
\end{array}\right)\left(i^{m}\right)^{n}
$$

Por lo cual los patrones de malabarismo son combinaciones de lanzamientos validados por un sistema de notación matemático llamado permutación de sitios, lo cual vincula cada objeto lanzado con el tiempo que permanece en el aire, tiempo que se mide en pulsos o segundos. Por ejemplo, un lanzamiento con un solo pulso significa que el malabarista se limita a pasar la pelota de una mano a la otra. Si la pelota es arrojada al aire, la altura que alcanza determina cuánto tarda en volver a la mano del malabarista, si son dos pulsos, tres o más. Cuantos más pulsos, más arriba hay que lanzar la bola para que se mantenga el patrón (Butler et al., 2015).

Lo anterior se encuentra estrechamente vinculado con las competencias del bloque I para segundo grado de secundaria en México en tres ejes temáticos: sentido numérico y pensamiento algebraico a partir de patrones numéricos o ecuaciones; forma, espacio y medida mediante el estudio de las características de las figuras y trayectorias de objetos, así como en el manejo de información matemática mediante análisis y representación de datos y su relación con las nociones de probabilidad. En específico se trabajan los siguientes aprendizajes esperados: resolver problemas que implican el uso de las leyes de los exponentes y de la notación científica, comparar cualitativamente la probabilidad de eventos simples, explicar la diferencia entre eventos complementarios mutuamente excluyentes e independientes y representar sucesiones numéricas mediante una regla dada (SEP, 2011).

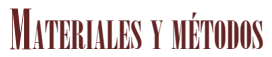

Pecora y Casey (2016) señalan que la investigación educativa de tipo experimental con población infantil implica una intervención con usuarios en entornos menos restrictivos que estén diseñados acorde a lo establecido en la intervención psicopedagógica. Se requiere de una planificación con la cantidad mayor de intervenciones efectivas y su principal desafío está en definir mejor los métodos basados en evidencia empírica, con mayores tiempos y controles durante la selección de usuarios, así como validez de las técnicas de entrenamiento implementadas. 
La investigación se realizó en la Unidad Técnico-Experimental en Fonoaudiología, Semántica-Léxica y Didáctica Musical de la Universidad Autónoma de Ciudad Juárez (UACJ). Con apoyo de seis practicantes del Programa de Licenciatura en Educación que atendieron la clase matemáticas en dos grupos de segundo año en una escuela secundaria de Nuevo Casas Grandes, Chih., durante el ciclo escolar 20172018. Se contó con el consentimiento informado de las familias.

Se trabajó con 47 adolescentes en edad promedio de 13 años y sin experiencia previa de entrenamiento circense que iniciaban el segundo año de educación secundaria. El adiestramiento en las malabares tuvo una duración de seis meses y se involucró la participación de seis practicantes que previamente fueron capacitados por el autor y coautoras del artículo. Se contó con el apoyo corresponsable del docente de matemáticas que impartía cuatro grupos de segundo de secundaria. Esto permitió que la investigación separase dos grupos experimentales (grupo 1 y grupo 2) con un total de 47 adolescentes; 23 aprendices fueron entrenados en las malabares con base en el método Rehoruli desarrollado por el malabarista alemán Stephan Ehlers (Ehlers, 2014) y 24 aprendices recibieron el entrenamiento de los patrones de malabares descritos por Rupert Ingalese en 1921 (citado en Ball Juggling, 1998). Además, se conocieron los resultados de aprovechamiento mediante la calificación final obtenida en la clase de matemáticas al cierre del ciclo escolar 2017-2018 y estos fueron contrastados con el promedio grupal de dos grupos control (grupo 3 y grupo 4) sin entrenamiento que incluían un total de 51 adolescentes.

Se entregó un paquete de tres pañuelos a cada aprendiz y se trabajó 15 minutos en la clase de matemáticas con una frecuencia de dos veces por semana y la dificultad de los ejercicios de malabares aumentaba cada semana. La familia recibió orientación en las artes malabares para que el adolescente realizará calentamientos y ejercicios por 10 o 20 minutos diarios en casa. En cada sesión se registró el tiempo de ejercicio y los números de lanzamientos exitosos, así como la frecuencia media de lanzamientos en cada grupo.

El efecto del entrenamiento de malabares en la evaluación de las funciones cognitivas se realizó con el empleo del software de malabarismo conocido como Juggling Lab diseñado por Boyce (2014), además de la aplicación de pruebas individuales para cada adolescente con ítems de patrones de malabares visuales, tareas de rotación mental con símbolos a diferentes grados de rotación e identificación de vértices, lados y caras planas en figuras tridimensionales en una computadora portátil. La evaluación final de la investigación también implicó esperar al mes de julio 2018 para conocer la nota final alcanzada en la clase de matemáticas por cada adolescente en el ciclo escolar 2017-2018.

\section{Ressurtunos}

Los dos grupos experimentales (grupo 1 y grupo 2) tuvieron resultados similares sobre las habilidades motrices al alcanzar tareas de precisión con tres pelotas para lanzar y atrapar en las tres figuras propuestas por Rupert Ingalese para distintas al- 
Figuras 1, 2 y 3. Patrones de malabares descritos por Rupert Ingalese en 1921.

Fuente: Ball Juggling (1998).
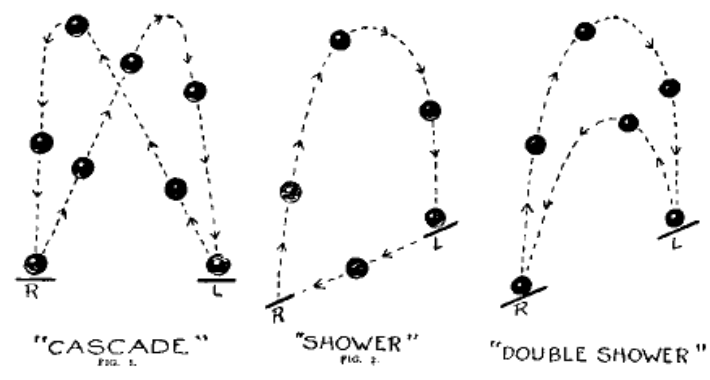

turas: cascade (fig. 1), shower (fig. 2) y double shower (fig. 3), así como al modelar matemáticamente el patrón de malabares e interpretarlo gráficamente.

El grupo experimental con el método Rehoruli (grupo 1) logró estar en promedio siete puntos más arriba que el otro grupo experimental con el método de Rupert Ingalese (grupo 2); es decir, siente aprendices más del grupo 1 -a saber 11 adolescentes contra cuatro del grupo 2- lograron desarrollar sus habilidades de disociación al poder practicar una transición entre las figuras cascade y shower sin detener las bolas.

Ambos grupos experimentales lograron al final de las sesiones de entrenamiento las acciones motrices al manipular pañuelos y pelotas en el aire: lanzar y recibir, equilibrar objetos sobre alguna parte del cuerpo, enganchar un objeto bajo presión con ayuda de otros objetos o partes del cuerpo, malabarismos de contacto, así como realizar toques o golpes precisos para proyectar los objetos y balanceos adecuados para generar las figuras correctas.

Al inicio de la investigación, y antes de los entrenamientos con las malabares con pañuelo y con pelotas, el autor aplicó pruebas individuales para cada adolescente con ítems de patrones de malabares visuales, tareas de rotación mental con símbolos a diferentes grados de rotación e identificación de vértices, lados y caras planas en figuras tridimensionales en una laptop a una muestra de 40 adolescentes (10 adolescentes por cada grupo de segundo año de la escuela secundaria). No se encontraron diferencias significativas en los resultados en promedio por grupo entre las respuestas de estos 40 adolescentes (tabla 1).

Después del entrenamiento, ambos grupos experimentales mejoraron su rendimiento respecto a la rotación mental (tabla 2), lo que permite evidenciar que las rotaciones manuales que se realizan con las malabares pueden estar relacionadas

Tabla 1. Resultados de pruebas individuales al inicio

\begin{tabular}{lcccc}
\hline Prueba & Grupo 1 & Grupo 2 & Grupo 3 & Grupo 4 \\
\hline $\begin{array}{l}\text { Identificación y ejecución de los } \\
\text { patrones de malabares visuales. }\end{array}$ & $0 / 10$ & $0 / 10$ & $0 / 10$ & $0 / 10$ \\
\hline $\begin{array}{l}\text { Rotación mental de símbolos; definir si } \\
\begin{array}{l}\text { son similares o diferentes en menos de } \\
\text { un minuto. }\end{array}\end{array}$ & $2 / 10$ & $3 / 10$ & $2 / 10$ & $2 / 10$ \\
\hline $\begin{array}{l}\text { Identificación de vértices, lados y caras } \\
\text { planas en figuras tridimensionales. }\end{array}$ & $4 / 10$ & $3 / 10$ & $4 / 10$ & $4 / 10$ \\
\hline
\end{tabular}




\begin{tabular}{lcccc}
\hline \multicolumn{5}{c}{ Tabla 2. Resultados de pruebas individuales al final } \\
\hline Prueba & Grupo 1 & Grupo 2 & Grupo 3 & Grupo 4 \\
\hline $\begin{array}{l}\text { Identificación y ejecución de los } \\
\text { patrones de malabares visuales. }\end{array}$ & $10 / 10$ & $10 / 10$ & $0 / 10$ & $2 / 10$ \\
\hline $\begin{array}{l}\text { Rotación mental de símbolos; definir si } \\
\text { son similares o diferentes en menos de } \\
\text { un minuto. }\end{array}$ & $7 / 10$ & $8 / 10$ & $4 / 10$ & $4 / 10$ \\
\hline $\begin{array}{l}\text { Identificación de vértices, lados y caras } \\
\text { planas en figuras tridimensionales. }\end{array}$ & $9 / 10$ & $8 / 10$ & $6 / 10$ & $5 / 10$ \\
\hline
\end{tabular}

con los sistemas sensoriales y de acción de las rotaciones mentales. Por otro lado, el entrenamiento en los malabares y el uso de la aplicación Juggling Lab permitieron que las y los adolescentes de ambos grupos experimentales mejoraran en promedio sus tareas de identificar y ejecutar patrones numéricos en imágenes visuales de malabares, en los mismos tiempos de respuesta ante símbolos similares con diferentes grados de rotación y también al responder correctamente a preguntas sobre figuras tridimensionales.

Por último, el promedio de los dos grupos experimentales en la clase de matemáticas fue de 9.25 y el promedio de los dos grupos control fue de 8.75. Por esto es posible indicar que la práctica constante de las malabares basada en ambas metodologías de enseñanza mejora el aprovechamiento de adolescentes de secundaria en la clase de matemáticas del segundo año escolar y también desarrolla habilidades de pensamiento matemático en un clima de aprendizaje con actividades lúdico-creativas y en apoyo de las disciplinas del modelo STEM.

\section{C'oncussiós}

La investigación permitió ganar habilidades de innovación en la praxis pedagógica del profesorado para el empleo de la pedagogía de las malabares en la clase de matemáticas en segundo año de secundaria. También para considerar su aplicación en las clases de ciencia, tecnología e ingeniería dentro del modelo STEM. La participación consciente de los padres de familia como facilitadores durante todo el proceso de entrenamiento. Las experiencias creativas que resultaron a partir de las sesiones de entrenamiento en malabares con adolescentes profundizaron en los referentes matemáticos e incluso geométricos que permiten explicar los patrones de malabarismo. La realización del estudio fue muy elogiada por el profesor de matemáticas y por la dirección de la escuela secundaria, quienes vieron en la pedagogía de las malabares una oportunidad para mejorar el aprovechamiento en la clase de matemáticas, el interés por las artes circenses, la motivación ante el estudio, las actitudes hacia la clase, las habilidades cognitivas y la integración de las y los adolescentes, por lo cual se piensa en replicar la investigación en otros centros escolares.

Se definieron algunos temas centrales para mejorar los programas de capacitación de docentes de matemáticas en escuelas secundarias en relación con el modelo 76 STEM: desarrollo práctico de la pedagogía del malabarismo con docentes y estu- 
diantes de secundaria; exploración y enumeración de patrones malabares simples y múltiples; automatización de las secuencias malabares con apoyo de la música de circo y el manejo correcto de las acciones motrices propias de la manipulación de objetos en el aire; empleo del software de malabarismo en el diseño de nuevos modelos matemáticos que expliquen patrones de malabares complicados dentro de la aplicación Juggling Lab.

\section{ReFreneritIs}

Adoevtseva, I. (2017). Características del área de la asignatura de enseñanza de ARTE (Música, Artes Visuales). Departamento de Educación y Ciencia de la región de Kostroma, 1-24. Rusia: Departamento de disciplinas humanitarias y artísticas y estéticas. Recuperado de: http://www. eduportal44.ru/

Alcántara, A. (2012). El formador del circo social. Revista semestral para educadores y animadores sociales, 12(1), 1-9. España: Universidad Autónoma de Barcelona.

Ayyer, A., Bouttier, J. y Corteel, S. (2015). Multivariate juggling probabilities. Department of Mathematics, Indian Institute of Science, 3(1), 1-28. India: Projet Émergences Combinatoire. Recuperado de: https://arxiv.org/pdf/1402.3752.pdf

Ball Juggling (1998). Juggling- Rupert Ingalese in 1921. Estados Unidos: Juggling Information Service. Recuperado de: http://www.juggling.org/books/ingalese/chap2. html

Boyce, J. (2014). Juggling Lab. Aplicación para crear patrones de malabares animados. Estados Unidos: Este software es una aplicación del GNU General Public License/ Source Forge. Recuperado de: http://jugglinglab.sourceforge.net/

Butler, S., Chung, F., Cummings, J. y Graham, R. (2015). Juggling card sequences. Computer Science and Engineering, 3(1), 1-29. Estados Unidos: Dept. of Computer Science and Engineering, UC San Diego. Recuperado de https://arxiv.org/ pdf/1504.01426.pdf

Czedli, G. (2013). On the mathematics of simple juggling patterns. Within Budapest Semester of Mathematics. Budapest Semester of Mathematics, 1-9. Estados Unidos: Conference populars. Recuperado de: http://www.math.u-szeged.hu/ czedli/ conference-talks/populars/juggling2013april19-slides.pdf

Echeverry, F. y Audor, Y. (2013). La matemática en los malabares. Revista Científica, 12(1), 303-307. Colombia: Universidad del Valle. Recuperado de: http://revistas. udistrital.edu.co/ojs/index.php/revcie/article/view/7059/8729

Ehlers, S. (2014). Aprender a hacer malabares con Jongloro, 1-33. Marvin Clifford (Ilustrador) y Peter Rupprecht (Traductor). Alemania: FQL Publishing. Recuperado de: http://fql-publishing.com/1---Leseprobe JmJ spanisch.pdf

Engström, A., Leskelä, L. y Varpanen, H. (2013). Geometric juggling with q-analogues. Department of Mathematics and Systems Analysis, 7(1), 1-13. Finlandia: University of Jyvaskyla. Recuperado de: https://www.researchgate.net/publication/257592288_ Geometric juggling with -analogues

Golant Media Ventures (2015). The adoption of digital technology in the arts. Digital Innovation Fund for the Arts in Wales, 1-33. Estados Unidos: Evidence Review. Recuperado de: https://www.nesta.org.uk/sites/default/files/difaw_gmv_e.pdf

Guerrero, J. (2017). Percepción del campo profesional en educación física. Revista educación y ciencia, 48(6), 23-45. México: Consejo nacional de investigación. Recuperado de: http://www.comie.org.mx/congreso/memoriaelectronica/v09/ ponencias/at06/PRE1177999880.pdf 
Guide pratique de l'École de cirque en France (GPECF, 2012). Les activités des arts du cirque. Francia: Fiche pro culture.

Guzmán, N., Moral, M. y Torres, G. (2015). Técnicas circenses en Educación Física en Infantil. Aplicación práctica. Trances, 7(2),147-160. España: Universidad de Jaen. Recuperado de: http://www.trances.es/papers/TCS\%2007 2_1.pdf

Jansen, P., Lange, L. y Heil, M. (2011). The influence of juggling on mental rotation performance in children. Biomedical Human Kinetics, 3(1), 18-22. Alemania: University of Düsseldorf. Recuperado de: https://www.researchgate. net/publication/271051920 The influence of juggling_on_mental_rotation_ performance

Kreilinger, B. (2015). Künstlerische Bildung an den Volkshochschulen. Kreativität und Gestalten bilden eine wichtige Säule im Programm der VHS, 1-10. Rusia: Bildung wrikt. Recuperado de: https://erwachsenenbildung.at/aktuell/nachrichten_details. php?nid=8034

Mauclair, D. (2003). Historia del circo. Viaje extraordinario alrededor del mundo. España: Editorial Milenio.

Ouellette, J. (2013). The Mathematics of Juggling. Quanta Magazine, 17(1), 1-12. Estados unidos: Newsletter. Recuperado de: https://www.quantamagazine.org/themathematics-of-juggling-20170524/

Palominos, A., Díaz, D., Jaña, N., Carreño, A. y Soto, V. (2018). Pedagogía del Malabarismo. Herramienta educativa que potencia el desarrollo integral. Chile: Colaboradores: Ed. Cuatro manos y Cabeza de Martillo. Con apoyo del Consejo Nacional de la Cultura y las Artes del Gobierno de Chile/ FONDART, Conv. 2017.

Pecora, P. y Casey, D. (2016). Elements of Effective Practice for Children and Youth Served by Therapeutic Residential Care. Safe strong supportive, 12(1), 1-82. Estados Unidos: Research Brief. Recuperado de: https://www.casey.org/media/Group-Carecomplete.pdf

Polster, B. (2015). The Mathematics of Juggling. Scientific Aspects of Jugging, 12(1), 1-24. Estados Unidos: Shannons. Recuperado de: https://www.qedcat.com/articles/ juggling survey.pdf

Price, Ch. (2012). Circus for Schools: Bringing a Circo Arts Dimension to Physical Education. Revue Phenps, 4(1), 1-9. Nueva Zelanda: University of Canterbury. Recuperado de: http://ojs.acadiau.ca/index.php/phenex/article/view/1446/1230

Programa para el Desarrollo Profesional Docente (PRODEP, 2018). Cuerpos Académicos Reconocidos. Recuperado de: https://promep.sep.gob.mx/cal/

Ruiz, C. y Mota, M. (2009). Programa de educación artística, cultura y ciudadanía. Organización de Estados Iberoamericanos para la Educación la Ciencia y la Cultura (OEI)

Secretaría de Educación Pública (2011). Programa de Estudios 2011. Guía para el Maestro. Educación Básica. Secundaria. Matemáticas. México: Gobierno Federal/ DGDC y DGFCN. Recuperado de: http://www.excelduc.org.mx/sys-uploads/ documentos/programas_de_estudio_2011._secundaria._matematicas.pdf

The National Advocates for Arts Education (NAAE, 2017). Submission to the Inquiry into innovation and creativity: workforce for the new economy. Australian Society for Music Education. Recuperado de: https://eprints.qut.edu.au/108973/1/ NAAEsubmission-Inquiry\%20into\%20innovation $\% 20$ and $\% 20$ creativity-Feb2017FINAL.pdf

Turner, R., Dubois, J. y Moris, S. (2016). Research on youth and social circus pedagogy. Chaning lives through circus, 1-148. Francia: Haute Ecole Leonard de Vinci. Recuperado de: http://sirkusinfo.fi/hallinta/wp-content/uploads/2016/05/CaravanCircus.pdf 